\title{
Nonlinear Spatio-Temporal Dynamics in Semiconductors
}

\author{
Eckehard Schöll \\ Technische Universität Berlin, \\ Institut für Theoretische Physik, \\ Hardenbergstr. 36, D-10623 Berlin, Germany \\ schoell@physik.tu-berlin.de
}

Received 27 May, 1999

\begin{abstract}
We review recent theoretical advances in the modeling and computer simulation of complex nonlinear spatio-temporal dynamics of charge carriers in semiconductors. Among the particularly instructive examples are investigations of current filaments in doped semiconductors in the regime of low temperature impurity breakdown. The nascence of current filaments in thin GaAs films with different contact geometries, including circular samples (Corbino disks) with symmetry breaking instabilities, is investigated, and the role of global couplings induced by the operating load circuit is analysed.
\end{abstract}

Semiconductors are complex nonlinear dynamic systems which exhibit current instabilities and selforganized formation of spatio-temporal patterns under the influence of strong electric fields $[1,2,3,4,5]$. Within the past years semiconductors have become a convenient model system for the study of such phenomena since sophisticated growth technologies and spatially and temporally resolved measurement techniques have provided the possibility to investigate semiconductor structures with desired properties. While a description of transport in terms of simple reaction-diffusion or drift-diffusion models is possible for various semiconductor structures like pnpn-diodes, pin-diodes, or thin-film structures, some semiconductor systems may require refined modelling in terms of more sophisticated transport models like hydrodynamic balance equations for the carrier densities, mean carrier momenta and mean carrier energies, or even a kinetic approach in terms of the semiclassical Boltzmann equation or a full quantum transport theory [6]. Often a combination of these approaches is used.

Semiconductor transport instabilities are characterized by a nonlinear dependence of the current density j upon the electric field $\mathbf{E}$. This relation is often nonmonotonic and displays negative differential conductivity $(N D C)$. Two important cases are described by an $N$-shaped or an $S$-shaped $j(\mathcal{E})$ characteristic, and denoted by $N N D C$ and $S N D C$, respectively. However, more complicated forms like $Z$-shaped, loop-shaped, or disconnected characteristics are also possible [7]. In case of $N N D C$, the $N D C$ branch is often but not always - depending upon exernal circuit and boundary conditions - unstable against the formation of field domains, while in the $S N D C$ case current filamentation generally occurs [8]. These primary selforganized spatial patterns may themselves become unstable in secondary bifurcations leading to periodically or chaotically breathing, rocking, moving, or spiking filaments or domains, or even solid-state turbulence and spatio-temporal chaos.

Within the last decade the introduction of concepts and methods from nonlinear dynamics and pattern formation has stimulated a large amount of experimental and theoretical work in a variety of semiconducting materials, e.g. p-Ge or n-GaAs at liquid Helium temperatures, or layered semiconductor structures like pin, pnpn, double-barrier resonant tunneling or heterostructure hot electron diodes. Recent progress has been obtained in elaborate experimental techniques to detect spatially and temporally resolved structures like scanning electron microscopy $[9,10]$, scanning laser microscopy [11, 12, 13], potential probe measurements $[14,15]$, or quenched photoluminsescence [16]. Detailed computer simulations in one and two spatial dimensions have enabled a quantitative comparison between theory and experiment. While in earlier work irregular and chaotic temporal behavior was in the cen- 
ter of interest $[17,18]$, the focus has now shifted towards more complex spatio-temporal dynamics including the dynamics of solitary filaments and multifilamentary states, spatio-temporal chaos, higher bifurcations of the elementary dissipative structures, interaction of defects and structural imperfections with pattern formation, and complex two-dimensional sample and contact geometries.

In this review some of these concepts will be illustrated by the dynamics of current filaments in the regime of low-temperature impurity breakdown in nGaAs where SNDC occurs. S-shaped characteristics and current filamentation have also been observed in heterostructure hot electron diodes [19, 20, 21] and in thyristor-like multilayered $\mathrm{Si}$ or GaAs semiconductor structures [22, 23, 24]. Other semiconductor systems where nonlinear transport phenomena and pattern formation have been found include parallel transport in modulation-doped heterostructures associated with real-space transfer [25, 26], i.e., the real-space analog of the Gunn effect, and vertical transport in a superlattice $[27,28,29,30]$, as well as field-enhanced trapping in semi-insulating GaAs [31]; in all those systems the current-field characteristic is $\mathrm{N}$-shaped and leads to the formation of electric field domains and oscillatory instabilities. Nonlinear spatio-temporal carrier dynamics in low-dimensional semiconductor structures like quantum wires and quantum dots has also become an active field recently $[32,6]$.

\section{Simulation of current fila- ments in the impurity break- down regime}

Impact ionization of charge carriers is a key process in inducing instabilites and spatio-temporal structures in semiconductors. It is a process in which a charge carrier with high kinetic energy collides with a second charge carrier, transferring its kinetic energy to the latter which is hereby lifted to a higher energy level. The result of the process is carrier multiplication which may induce electrical instabilities at sufficiently high external electric fields. Impact ionization from shallow donors or acceptors is responsible for impurity breakdown at low temperatures where almost all carriers are bound to shallow impurities. Being a nonlinear autocatalytic process, it induces a nonequilibrium phase transition [1] between a low conductivity and a high conductivity state and may lead to a menagery of spatio-temporal instabilities including self-oscillations and chaos as first observed by Aoki [33] and Teitsworth et al [34]. If a critical threshold voltage is applied to the semiconductor sample, the free carrier concentration grows in an avalanche-like manner, and the current increases by orders of magnitude at an almost constant voltage due to current filamentation and lateral growth of filaments.

Current filamentation is usually attributed to the bistability of an $S$-shaped dependence of the current density $j$ on the electric field $\mathcal{E}$, i.e. SNDC. The spatial coexistence of the high-conductivity and the lowconductivity state at a fixed voltage leads to the formation of current filaments. In generation-recombination models [1] the bistability is caused by impact ionization of multilevel impurities. The generation-recombination cycle starts with impact ionization from the ground state and is afterwards sustained by impact ionization of much less strongly bound excited states.

In the past, most theoretical work has been restricted to one-dimensional treatments. Breathing filaments [35, 36] and the intermittent and chaotic behavior of laterally traveling filaments [37, 38] have been studied in one-dimensional simulations where only the transverse spatial coordinate perpendicular to the current flow was taken into account. The spatio-temporal modes and the stochastics of the breakdown process have been investigated in a one-dimensional longitudinal model for $\mathrm{p}-\mathrm{Ge}$ [39]. Longitudinally travelling charge density waves have been found in simulations for n-GaAs [40]. The dipolar electric field between two point contacts was included in a phenomenological, effectively one-dimensional model for current filaments in n-GaAs [41]. Much progress has been made in the microscopic analysis of low-temperature impurity breakdown in terms of single-particle [42] and many-particle Monte Carlo (MC) simulations [43] for $\mathrm{p}-\mathrm{Ge}$ and for n-GaAs [44, 45]. In order to study the nascence of current filaments the transverse and the longitudinal degrees of freedom have been combined for a realistic twodimensional sample geometry with appropriately modelled point contacts, and detailed microscopic information on the generation-recombination kinetics obtained from MC simulations has been included $[46,47,5]$. The widening of the filaments in a weak transverse magnetic field in the direction of the Lorentz force has also been simulated [48] and circular geometries (Corbino disks) have been studied, too $[49,50]$. 
We consider a doped semiconductor at temperatures of liquid Helium. In the following we shall assume n-type material; p-type semiconductors can be treated analogously. The carrier density in the conduction band, and hence the current density, is determined by the generation-recombination (GR) processes of carriers between the conduction band and the donor levels. The experimentally observed S-shaped current densityfield relation in the regime of impurity breakdown can be explained in terms of standard GR kinetics only if impact ionization from at least two impurity levels is taken into account [51]. Therefore we model the infinite hydrogenlike energy spectrum of the shallow donors by the ground state and an "effective" excited state close to the band edge. In this case the state of the system can be characterized by the spatial distribution of the carrier densities in the conduction band $n(\mathbf{r}, t)$ as well as in the impurity ground state and excited state $n_{1}(\mathbf{r}, t), n_{2}(\mathbf{r}, t)$, respectively, where $\mathbf{r}$ is the position vector and $t$ denotes time.

In the following we shall adopt an approach which combines Monte Carlo (MC) simulations of the microscopic scattering processes and GR kinetics with rate equations for the macroscopic spatio-temporal dynamics of the carrier densities which occurs on a much slower time-scale than the microscopic dynamics. First, we will list the macroscopic constitutive equations.

The temporal evolution of $n$ is governed by the continuity equation

$$
\dot{n}=\frac{1}{e} \nabla \cdot \mathbf{j}+\phi\left(n, n_{1}, n_{2},|\mathbf{E}|\right),
$$

where the dot denotes the partial derivative with respect to time, $e$ is the electron charge and $\mathbf{E}$ is the local electric field within the sample. Within the driftdiffusion approximation the current density $\mathbf{j}$ can be expressed as

$$
\mathbf{j}=e(n \mu \mathbf{E}+D \nabla n)
$$

with the field-dependent mobility $\mu$ and diffusion coefficient $D$. We assume the validity of the Einstein relation $D=\mu k_{B} T_{L} / e$, where $k_{B}$ is Boltzmann's constant and $T_{L}$ is the lattice temperature.

The rate $\phi$ of GR processes depends on the local values of the carrier densities in the conduction band and at the impurities, given by $n, n_{1}$, and $n_{2}$, respectively, and the strength of the electric field $\mathcal{E}=|\mathbf{E}|$. Analogously, rates $\phi_{1}, \phi_{2}$ determining the temporal evolution of $n_{1}, n_{2}$ can be defined as

$$
\dot{n}_{i}=\phi_{i}\left(n, n_{1}, n_{2}, \mathcal{E}\right)
$$

with $i=1,2$.

In an explicit two-level model [1], e.g., the GR rates are given by

$$
\begin{aligned}
\phi & =X_{1}^{S} n_{2}-T_{1}^{S} n p_{t}+X_{1} n n_{1}+X_{1}^{*} n n_{2} \\
\phi_{1} & =T^{*} n_{2}-X^{*} n_{1}-X_{1} n n_{1} \\
\phi_{2} & =-\phi-\phi_{1}
\end{aligned}
$$

where $p_{t}=N_{D}-n_{1}-n_{2}$ is the density of ionized donors, $N_{D}$ is the total density of donors, $X_{1}^{S}$ is the thermal ionization coefficient of the excited level, $T_{1}^{S}$ is its capture coefficient, $X_{1}, X_{1}^{*}$ are the impact ionization coefficients from the ground and excited level, respectively, $X^{*}, T^{*}$ denote the transition coefficients from the ground level to the excited level and vice versa, respectively.

The electric field is coupled to the carrier densities via Gauss' law

$$
\epsilon \nabla \cdot \mathbf{E}=e\left(N_{D}^{*}-n_{1}-n_{2}-n\right),
$$

where $\epsilon$ is the dielectric constant and $N_{D}^{*} \equiv N_{D}-N_{A}$ holds with the compensating acceptor concentration $N_{A}$. From equations (1),(3),(6),(7) the charge conservation equation can be derived, given by $\nabla \cdot \mathbf{J}=0$ with $\mathbf{J}=\epsilon \dot{\mathbf{E}}+\mathbf{j}$, where $\mathbf{J}$ is the total current density composed of displacement current and conduction current densities.

The essential nonlinearities of the constitutive model equations (1)-(7) in the regime of lowtemperature impurity breakdown are contained in the dependence of the GR coefficients upon $n, n_{1}, n_{2}$, and $\mathcal{E}$. In order to derive these from a microscopic theory we have performed single particle MC simulations for a spatially homogeneous steady state [44].

The microscopic rates of all band-impurity processes depend upon the carrier densities in the band and impurity states, which in turn depend upon the nonequilibrium carrier distribution function. To obtain these carrier densities, the MC method has to be combined self-consistently with the rate equations (1)-(3) in the homogeneous steady state, where the GR coefficients $X_{1}, X_{1}^{*}, T_{1}^{S}$ are calculated by averaging the microscopic transition probabilities $\left(P_{i i}^{1}, P_{i i}^{2}, P_{\text {rec }}\right.$ for impact ionization from the ground state, the excited state, and capture, respectively) over the nonequilibrium distribution function $f(\mathbf{k})$, which is extracted from the MC simulation at each step: 


$$
\begin{aligned}
& X_{1}\left(n, n_{1}, n_{2}, \mathcal{E}\right)=\frac{1}{n n_{1}} \int d^{3} k f\left(\mathbf{k} ; n, n_{1}, n_{2}, \mathcal{E}\right) P_{i i}^{1}\left(\mathbf{k}, n_{1}\right), \\
& X_{1}^{*}\left(n, n_{1}, n_{2}, \mathcal{E}\right)=\frac{1}{n n_{2}} \int d^{3} k f\left(\mathbf{k} ; n, n_{1}, n_{2}, \mathcal{E}\right) P_{i i}^{2}\left(\mathbf{k}, n_{2}\right), \\
& T_{1}^{S}\left(n, n_{1}, n_{2}, \mathcal{E}\right)=\frac{1}{n p_{t}} \int d^{3} k f\left(\mathbf{k} ; n, n_{1}, n_{2}, \mathcal{E}\right) P_{r e c}\left(\mathbf{k}, p_{t}\right) .
\end{aligned}
$$

Note that $f$, and hence $X_{1}, X_{1}^{*}$ and $T_{1}^{S}$, in turn depend parametrically on $n, n_{1}, n_{2}$ and $\mathcal{E}$. An iteration procedure, where $n_{1}$ and $n_{2}$ are expressed by their steadystate dependence on $n$ and $\mathcal{E}$, is used to solve the above problem self-consistently [44].

It is possible to express the dependence of the GR coefficients upon $\mathcal{E}$ and $n$ through the electron temperature $T_{e}(\mathcal{E}, n)[46]$. Here we use the notion of electron temperature $T_{e}$ in the usual sense of the mean electron energy $(3 / 2) k_{B} T_{e}=\langle E\rangle=\left(\hbar^{2} / 2 m\right)\left\langle k^{2}\right\rangle$, i.e. the electron temperature is extracted from the MC data essentially as the second moment of the nonequilibrium distribution function $f(\mathbf{k})$. This should not be confused with the concept of a heated Maxwellian distribution function, where the electron temperature appears as a parameter. This latter concept is much more restrictive since it assumes a quasi-thermal equilibrium of the conduction band subsystem. The MC data yield a strong increase of $T_{e}$ with rising electric field on the high-conductivity branch of the current-voltage characteristics as opposed to only a slight increase on the low-conductivity branch. Physically the strong increase is associated with a population inversion between the donor ground and the excited state on the upper branch. For $\mathcal{E}>9 \mathrm{~V} / \mathrm{cm}$ impact ionization does no longer contribute to energy relaxation in a significant way since the donor ground states are almost completely ionized and impact ionization from the excited state dominates, which is now more strongly populated than the ground state. The GR cycle runs now between the donor excited state and the conduction band. Because of the much smaller energies involved, this process cools less efficiently.

In the following sections our strategy will be to insert fitted analytical representations of the MC data into the macroscopic equations (1)-(7). We use this approach in order to take into account as much detailed information as possible about the microscopic scattering processes, while still retaining manageable expressions. As a check of consistency, the spatially uniform stationary $n(\mathcal{E})$ characteristic which we obtain with the fitting functions from (4)-(6) in the steady state using the condition of charge neutrality $N_{D}^{*}=$ $n(\mathcal{E})+n_{1}(\mathcal{E}, n)+n_{2}(\mathcal{E}, n)$, can be compared with the characteristic obtained by direct MC simulation. The $\mathrm{S}$-shaped negative differential conductivity (SNDC) is successfully reproduced.

\section{Current filament formation in point contact samples}

In this section we shall present exemplary twodimensional simulations for thin samples with point contacts, considering the longitudinal $x$-direction as well as the transverse $z$-direction. In this way it is possible to model the dynamics of current filaments. We consider a rectangular sample with side lengths $L_{x}=5$ and $L_{z}=7.5 \mathrm{~mm}$ with two small circular contacts of diameter $1 \mathrm{~mm}$ at a distance of $3.5 \mathrm{~mm}$ in $x$-direction, representing a thin $\mathrm{n}$-GaAs film with point contacts (thickness $14 \mu \mathrm{m}$ ). At the contacts $n$ is fixed to a value $n_{D}=5 \times 10^{15} \mathrm{~cm}^{-3}$ to model Ohmic contacts. All other boundaries are treated as insulating where the components of the current density $\mathbf{j}$ and the electric field $\mathbf{E}$ perpendicular to the boundaries vanish. Our numerical algorithm employs an implicit finite element scheme [52].

We study the nascence of current filaments when a bias voltage $U_{0}$ is applied to the sample in series with a load resistor $R$ so that the semiconductor is forced from the nearly insulating state to a highly conducting state. Within $1 \mathrm{ps}$ the bias voltage is linearly increased from $U_{0}=0$ to $U_{0}=20 \mathrm{~V}$. Initially the current density is very low (Fig. , $t=1 \mathrm{ps}$ ). Subsequently impact ionization multiplies the electron concentration at the high-field zones near the cathode and the anode and 
establishes two fronts that move towards each other $(\mathrm{t}=0.2 \mathrm{~ns})$. The propagation of the fronts is accompanied by a slightly increased electron temperature $T_{e}$. Although the electric field behind the front is smaller than ahead of it, for reasons of current continuity the increased electron density in regions passed by the front is almost conserved because recombination is a much slower process than generation. Hence impact ionization downstream is encouraged, whereas further generation upstream is inhibited. When the two fronts meet, a thin rudimentary filament is formed $(t=0.4 \mathrm{~ns})$. Now donor impact ionization is becoming enhanced in the rudimentary filament because the excited level $n_{2}$ is increasingly populated. The current density, the width and the electron temperature are significantly growing in the rudimentary filament. Finally impact ionization leads to a uniform increase of electron density until the filament reaches its mature state $(\mathrm{t}=1.4 \mathrm{~ns})$ where almost all donors are ionized, and the carrier density corresponds to the upper branch of the homogeneous steady state characteristic $n(\mathcal{E})$. Within the filament the excited donor level is much more highly populated than outside. Nevertheless still only about 2 percent of the band carriers are trapped in the excited level, while the ground level is completely depleted inside the filament. Thus the population ratio between ground and excited level is inverted in the filament. Also the current density $j$ and the electron temperature $T_{e}$ are much larger inside the filament than outside.

Fig. shows the fully developed stationary current filaments for five different values of a series load resistance $R$. At $R=100 \Omega$ the filament is very wide and plum-shaped. With increasing $R$ the operating point on the characteristic is shifted towards lower sample voltage and current, and the width of the filament decreases and finally the filament becomes very unstable and thin $(R=10 M \Omega)$. The change of the shape and the width of the filament describes a transition from a pattern induced by the dipolar electric field to a self-organized filament, in good agreement with experiments [16, 53, 5].

It has been found experimentally that an external magnetic field applied perpendicular to the electric field can sensitively affect the spatio-temporal instabilities in the regime of impurity impact ionization $[54,55,56]$. The dynamic Hall effect has been proposed as a mechanism to induce chaotic temporal os- cillations in the breakdown regime [57]. The transverse periodic or chaotic motion of a current filament under crossed electric and magnetic field has been numerically analyzed for p-type Ge by one-dimensional simulations based on the semiclassical semiconductortransport equations neglecting the longitudinal spatial dependence [38]. The spatial deformation of current filaments in the presence of a transverse magnetic field has been explained by an effectively one-dimensional drift-diffusion model assuming a dipole-like electric field between two point-contacts [41]. However, in order to fully understand the effects of a transverse magnetic field on the filament properties, two-dimensional analyses including the spatial dependence on both the longitudinal $x$ - and the transverse $z$-direction are indispensable [48].

The current density $\mathbf{j}(\mathbf{B})$ in the presence of a magnetic field $\mathbf{B}$ normal to the current flow is given by

$$
\mathbf{j}(\mathbf{B})=\frac{\mathbf{j}(0)+\mu \mathbf{B} \times \mathbf{j}(0)}{1+|\mu \mathbf{B}|^{2}},
$$

where $\mathbf{j}(0)$ is the current density for $B=0$ as given by Eq. (2).

Upon application of a voltage ramp and a magnetic field of a few $\mathrm{mT}$, first a thin, straight filament is formed as in the case without magnetic field. In the next stage, the straight filament is gradually widened and asymmetrically expanded in the direction of the Lorentz force [48]. The simulation shows that the electric fields at the filament border pointing in the direction of the Lorentz force are much stronger than at the opposite border. These asymmetric fields result from a superposition of the dipole field due to the space charge already present in the filament walls at $B=0$, and the Hall field. They are in good agreement with asymmetric ridge-like structures observed by scanning laser microscopy under a transverse magnetic field [11, 12, 41]. Also, the simulations and the experiments show that the width and curvature of the filaments become larger with increasing magnetic-field strength. Fig. shows the stationary current filaments under the action of a magnetic field for the same sample with two inner point contacts as in Figs. and. With increasing $B$ the filaments become asymmetrically distorted by the Lorentz force, and at large $B$ and large currrent they assume an S-shape as also found in experiments [58]. 


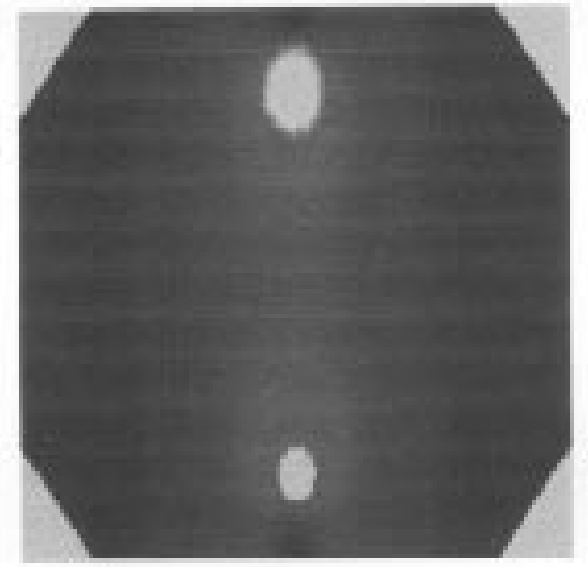

\section{$\mathrm{t}-1 \mathrm{ps}$}

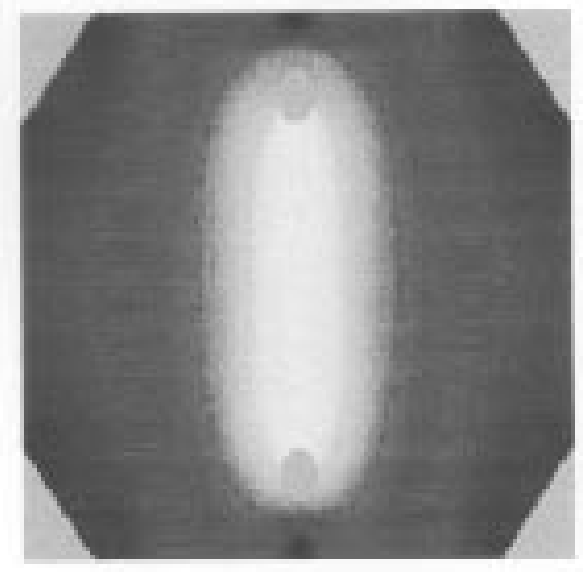

$\mathrm{t}=0.4 \mathrm{~ns}$

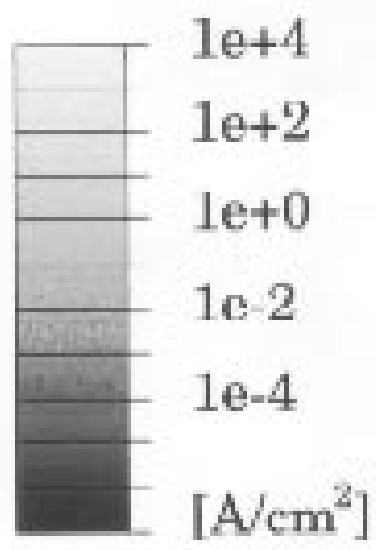

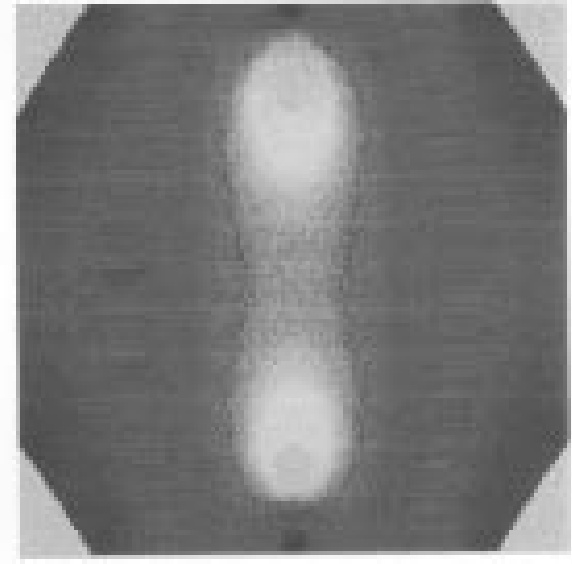

$\mathrm{t}=0.2 \mathrm{ng}$

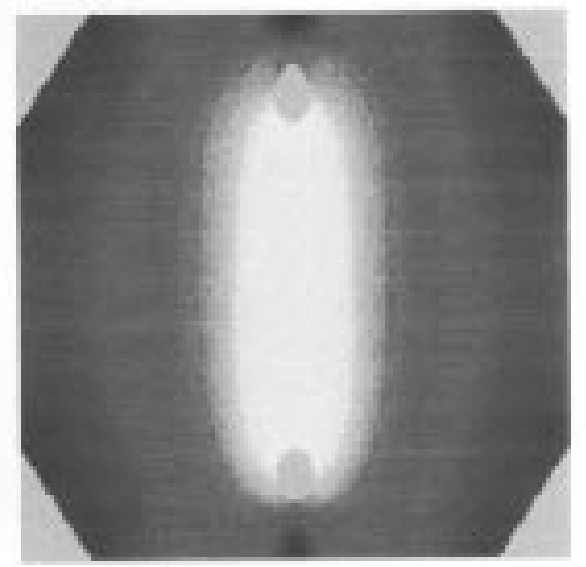

$\mathrm{t}=1.4 \mathrm{us}$

$\mathrm{U}_{0}=20 \mathrm{~V}$

$\mathrm{B}=0$

$\mathrm{R}=100 \mathrm{Ohm}$

Figure 1. Simulation of the formation of a current filament in n-GaAs. The temporal evolution of the current density $j(x, z)$ after application of a bias voltage $U_{0}=20 \mathrm{~V}$ via a load $R=100 \Omega$ is shown as a function of the spatial coordinates $x$ and $z$ for a rectangular sample with two inner point contacts for different times. Note that the injecting cathode is at the top (calculated for $T_{L}=4.2 \mathrm{~K}$ with the parameters $N_{D}=7.0 \times 10^{15} \mathrm{~cm}^{-3}, N_{A}=2.0 \times 10^{15} \mathrm{~cm}^{-3}, \mu=10^{5} \mathrm{~cm}^{2} / V_{s}, \epsilon_{r}=10.9$, and generation-recombination coefficients taken from a Monte Carlo simulation). 


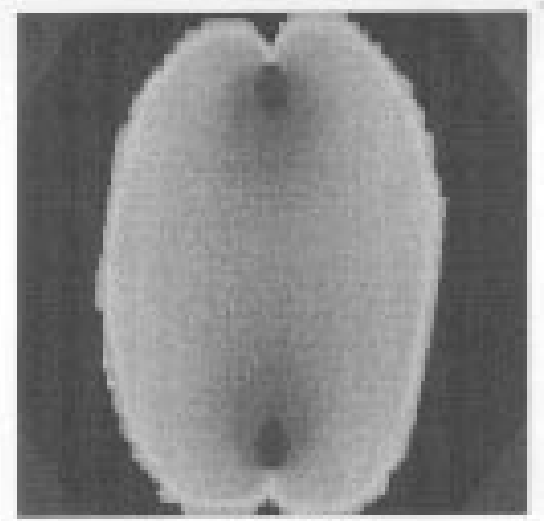

$\mathrm{R}-100 \mathrm{Ohm}$

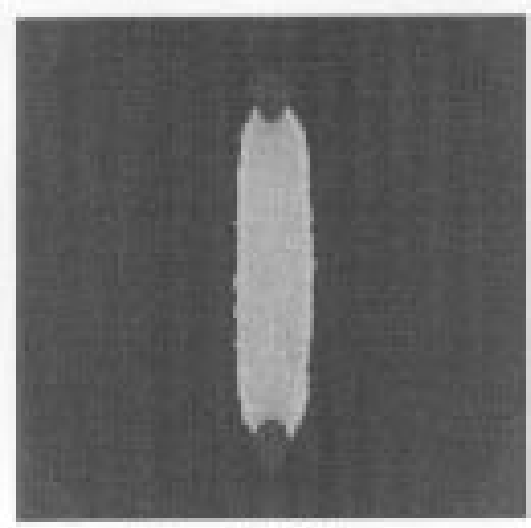

$\mathrm{R}=1 \mathrm{kOhm}$

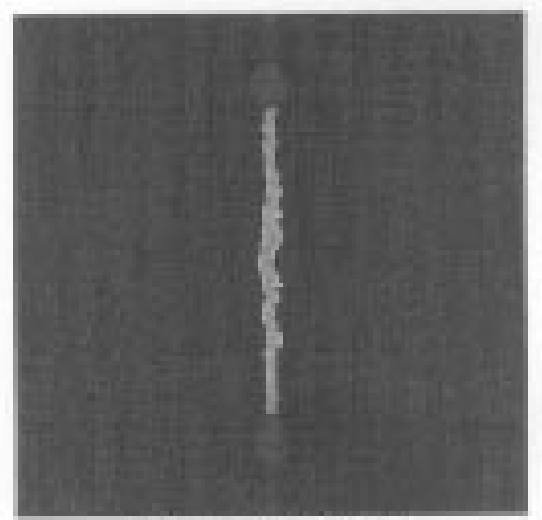

$\mathrm{R}-10 \mathrm{MOhm}$

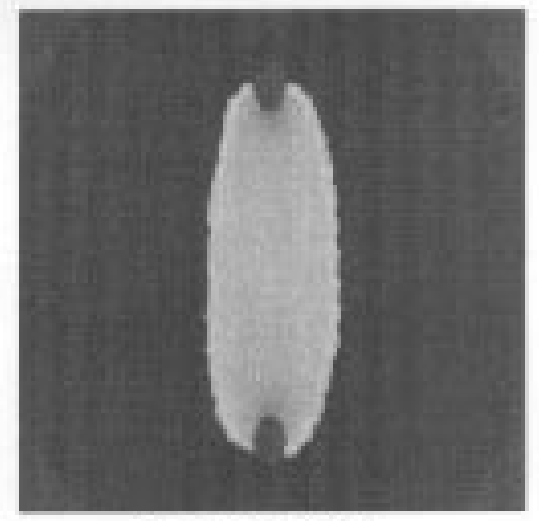

$\mathrm{R}=5000 \mathrm{hm}$

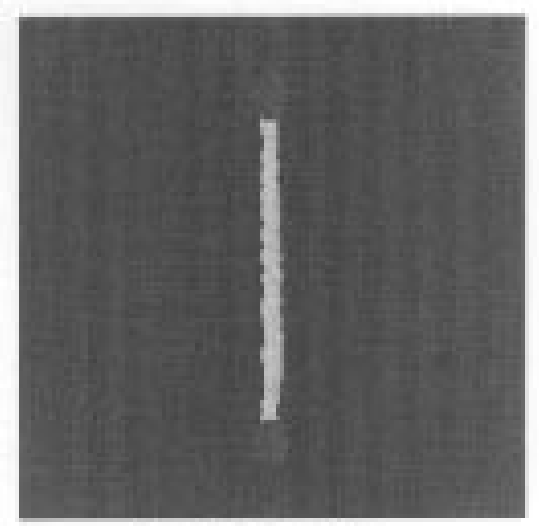

$\mathrm{R}=10 \mathrm{kOhm}$

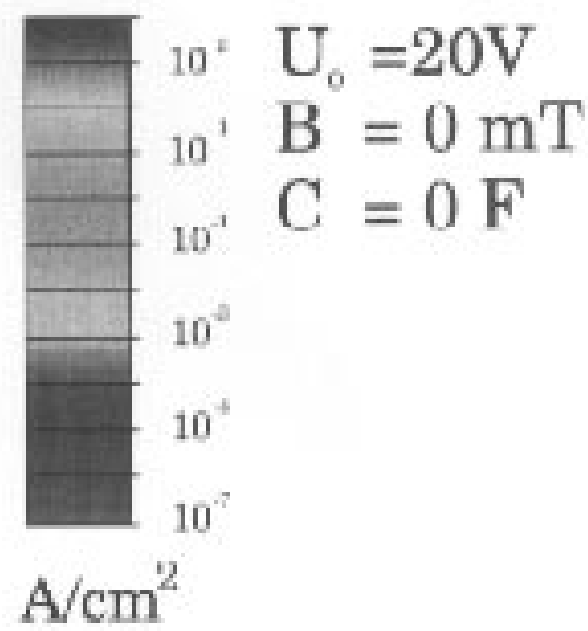

Figure 2. Simulated current filaments in a rectangular sample with two inner point contacts for an applied bias voltage $U_{0}=50 \mathrm{~V}$ and different load resistances $R$. The stationary current density $|\mathbf{j}(x, z)|$ is shown as a density plot. (calculated with the same parameters as Fig. .) 

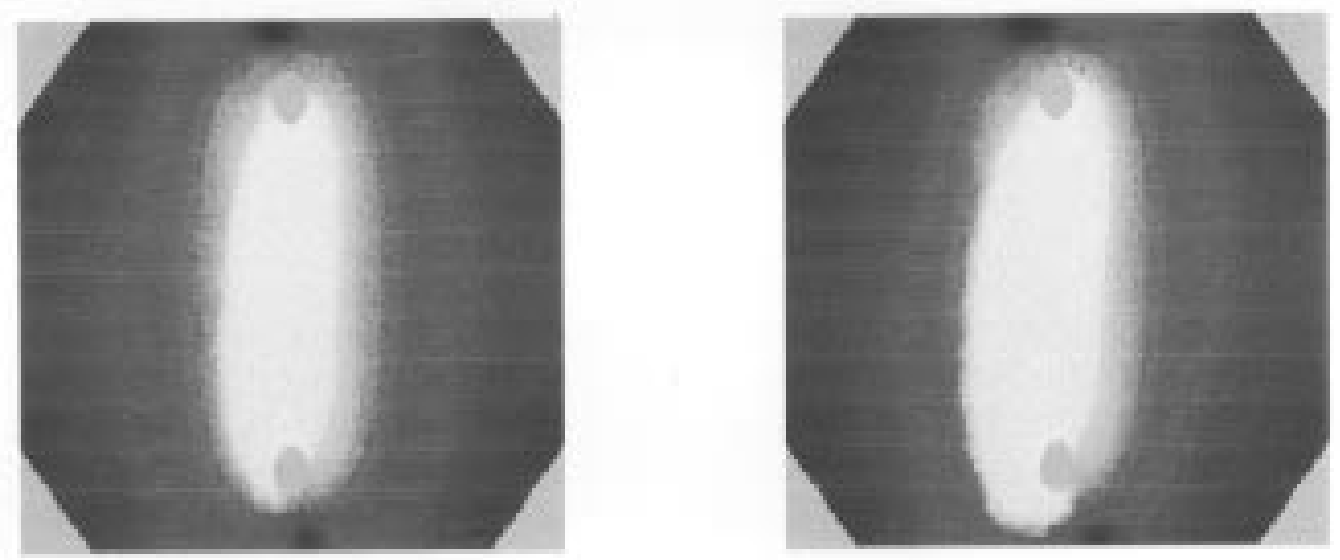

$\mathrm{B}=30 \mathrm{~m} T$

$\mathrm{B}=60 \mathrm{~m}^{\prime}$
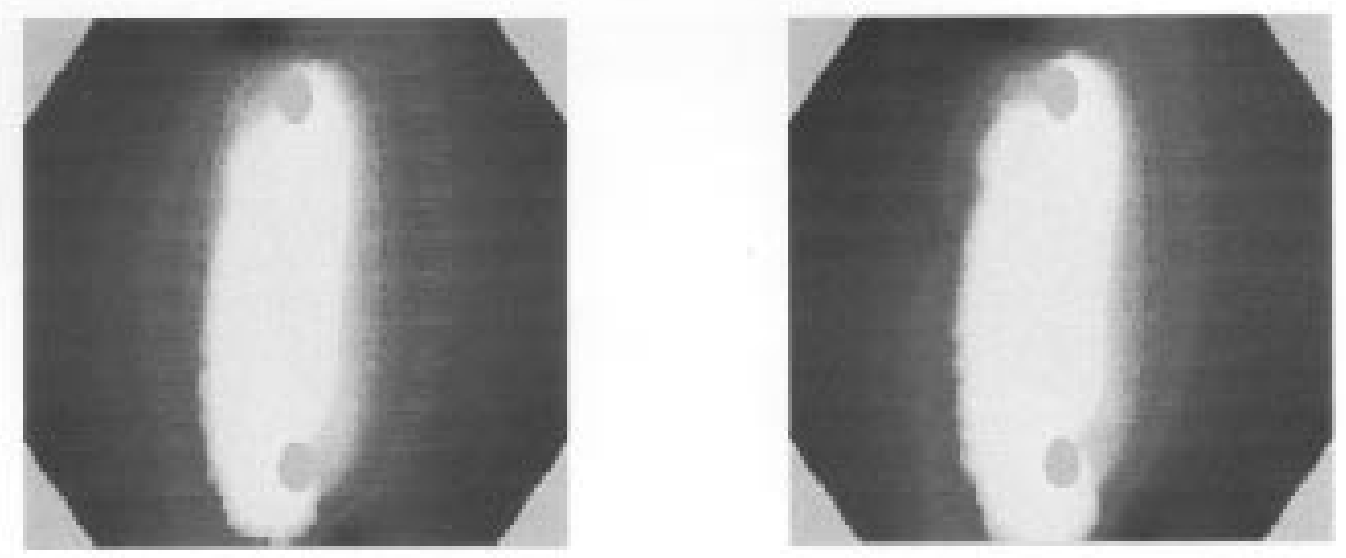

$\mathbf{B}=90 \mathrm{~m}^{\prime} \mathbf{l}^{\prime}$

$\mathrm{B}=120 \mathrm{mT}$

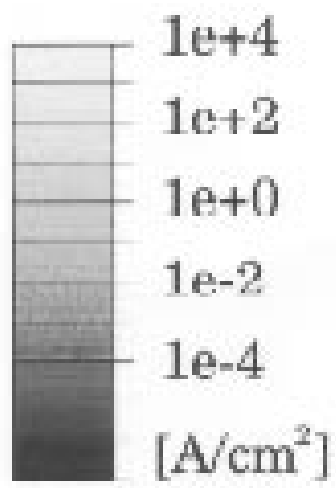

$$
\begin{aligned}
& \mathrm{U}_{0}=20 \mathrm{~V} \\
& \mathrm{R}=100 \mathrm{Ohm}
\end{aligned}
$$

Figure 3. Simulated current filaments in a rectangular sample with two inner point contacts for an applied bias voltage $U_{0}=20 \mathrm{~V}, R=100 \Omega$ and different perpendicular magnetic fields $B$. (calculated with the same parameters as Fig. .) 

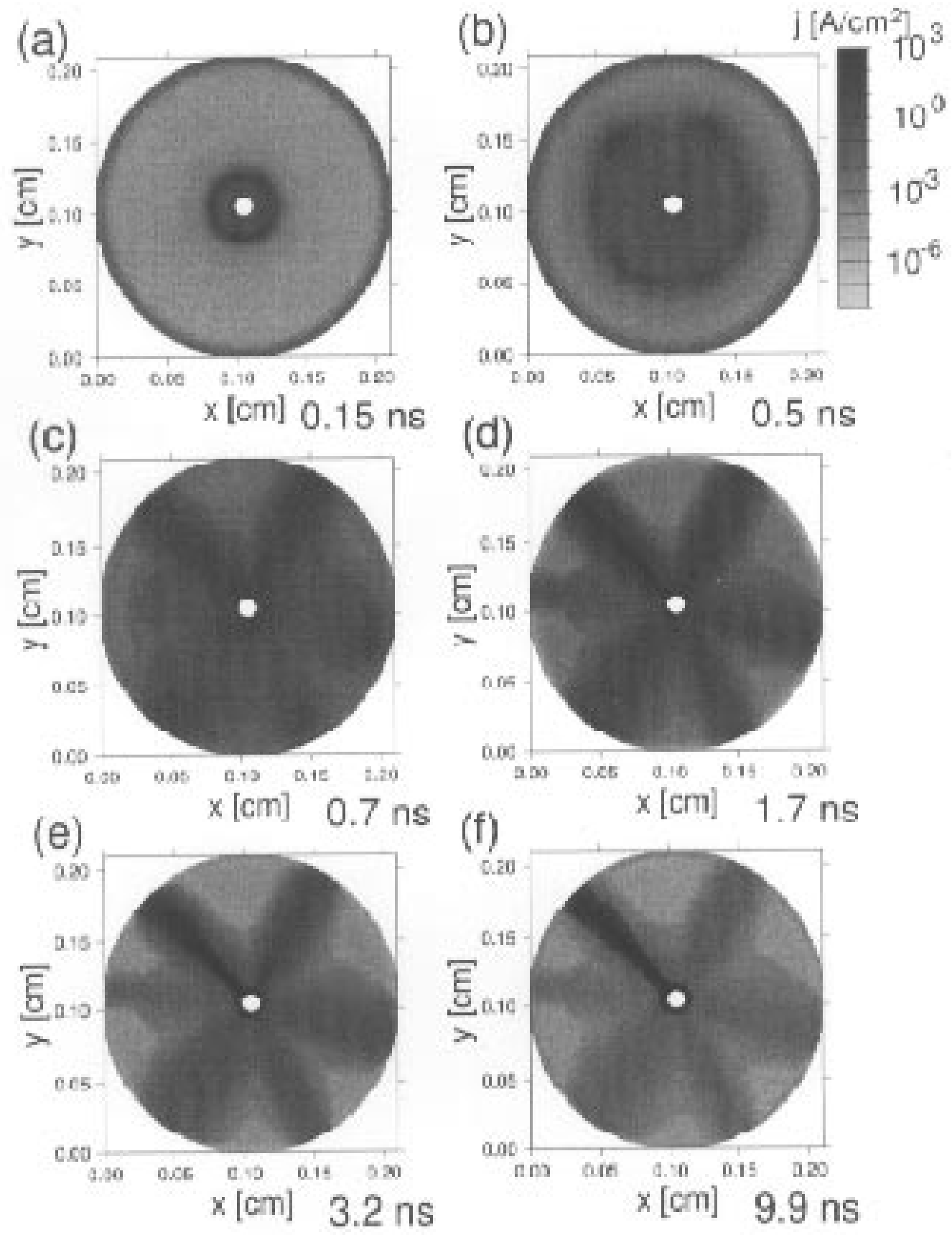

Figure 4. Simulation of the formation of a current filament in a Corbino disk. The temporal evolution of the current density $|\mathrm{j}(x, y)|$ is shown for a circular sample of diameter $2.1 \mathrm{~mm}$ and an inner contact diameter of $80 \mu \mathrm{m}$. a) $t=0.15 \mathrm{~ns}, \mathrm{~b}) t=0.5 \mathrm{~ns}$, c) $t=0.7 \mathrm{~ns}$, d) $t=1.7 \mathrm{~ns}$ e) $t=3.2 \mathrm{~ns}$ f) $t=9.9 \mathrm{~ns}$. The bias voltage $U_{0}=2.4 \mathrm{~V}$ and the load resistance $R=10 \mathrm{k} \Omega$ correspond to an operating point with an average field $\mathcal{E}_{a v}=4.7 \mathrm{~V} / \mathrm{cm}$, i.e., between the holding field $\mathcal{E}_{h}=4.0 \mathrm{~V} / \mathrm{cm}$ and the threshold field $\mathcal{E}_{t h}=17 \mathrm{~V} / \mathrm{cm}$ of the homogeneous $n(\mathcal{E})$ characteristic (calculated with the same parameters as Fig.). 


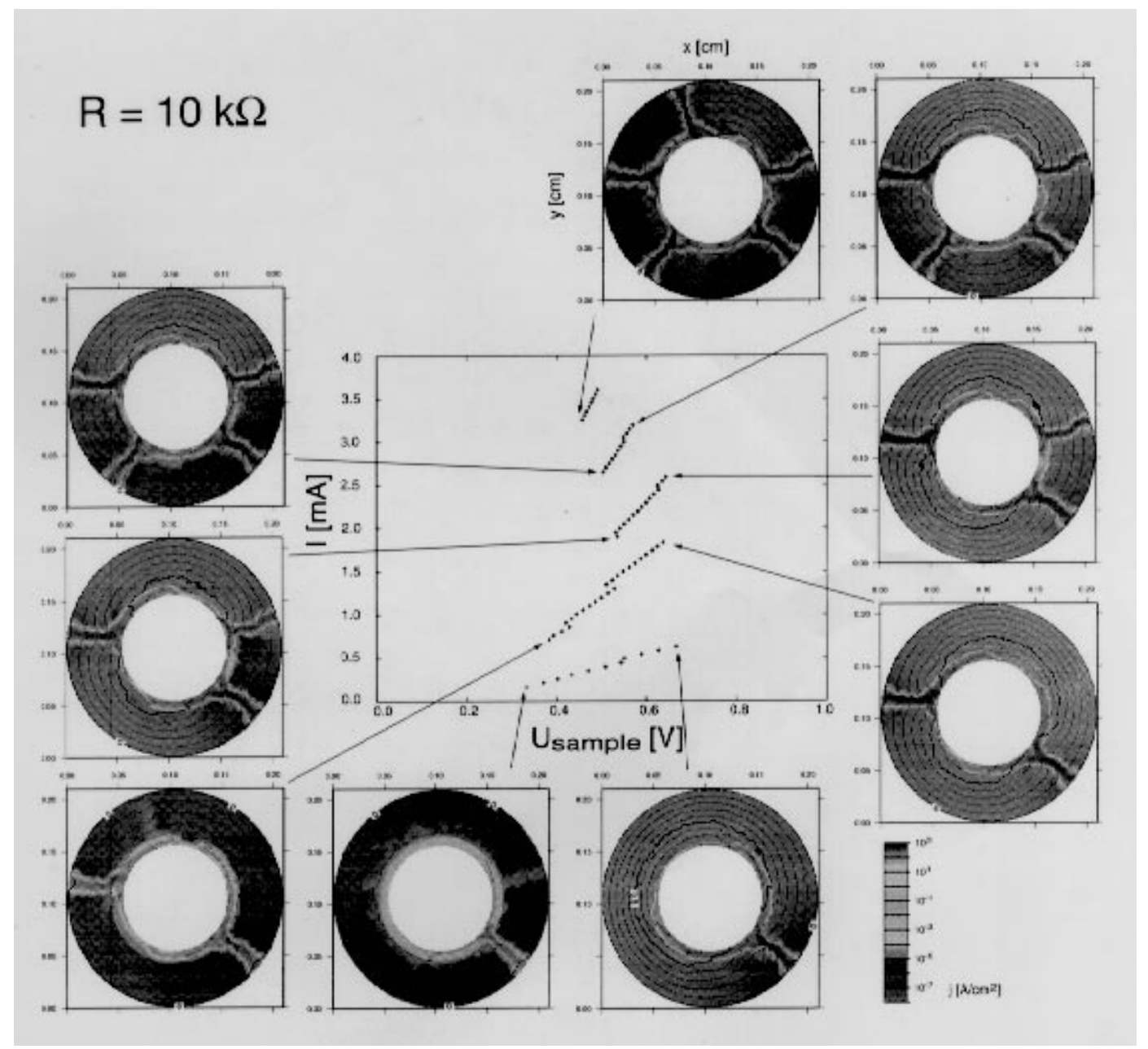

Figure 5. Simulated current-voltage characteristic of a circular sample with two concentric contacts of diameters 1 mm and $2.1 \mathrm{~mm}$. The insets show the current density $|\mathbf{j}(x, y)|$ for various operating points as indicated by arrows. The bias voltage $U_{0}$ is swept from 2.0 to $36.5 \mathrm{~V}$ and the load resistance is $R=10 \mathrm{k} \Omega$ (calculated with the same parameters as Fig.).

\section{Current filaments in Corbino disks}

Next, we present simulations of a sample with circular contact symmetry (Corbino disk). The sample is confined by an outer ring electrode of radius $1050 \mu \mathrm{m}$ (anode) and has a concentric inner point contact of $80 \mu \mathrm{m}$ diameter (cathode) in the center of the disk. Such geometries offer the possibility to study pattern formation in samples without lateral boundaries, and are therefore of considerable interest. Various self-organized patterns of single and multiple current filaments have been observed experimentally in such samples [59].

In our simulation a constant bias voltage $U_{0}=2.4 \mathrm{~V}$ is applied to the Corbino disk of thickness $3 \mu \mathrm{m}$ via a load resistor $R=10 \mathrm{k} \Omega$. Initially the sample is in the insulating state. Subsequently impact ionization multiplies the electron concentration at the injecting inner point contact and establishes a radially symmet- ric front that moves towards the circular anode (Fig. (a)). At a certain radius the circular front breaks up into several streamers (b), each of which, upon reaching the anode, forms a rudimentary radial current filament (c). The symmetry of this pre-filamentary state, i.e. the number of rudimentary filaments, depends on sample parameters as well as on the applied voltage. The pre-filaments then start growing in current while retaining approximately their original width $(\mathrm{d})$. This leads to a rising overall current $I$ and thus, via the external load resistance, which acts as a global constraint due to Kirchhoff's law $U_{0}=U+R I$, to a reduction in the sample voltage $U$. The pre-filaments therefore enter into a competition as a result of which only the filament which has first reached the outer contact survives (e). The other filaments slowly decay into the low-conducting state and vanish (f).

This winner-takes-all dynamics is characteristic of a global coupling as imposed by the load resistance, and 
is familiar from general nonlinear self-organizing processes under global constraints [60].

If the operating point is shifted to larger currents, more than one filament can survive. This is shown in Fig. where the bias voltage $U_{0}$ is increased from $2.0 \mathrm{~V}$ to $36.5 \mathrm{~V}$. The insets show the stationary current density distribution at different operating points for a sample with two concentric contacts with diameters $1 \mathrm{~mm}$ and $2.1 \mathrm{~mm}$. As the current increases, the current density in the filament grows up to a threshold where a second filament is formed. This decreases the sample resistance, resulting in a switch-back of the sample voltage according to Kirchhoff's law. Thus a second branch of the current-voltage characteristic arises, corresponding to two filaments. Similarly, successive branches with three, four, five, etc. filaments occur. Each jump in the current-voltage characteristic is associated with the generation of an additional filament. Hysteresis with respect to bias sweep-up and sweep-down is also found. Such behavior is in good agreement with experiments [59].

\section{Conclusions}

In this review we have presented concepts of spatiotemporal self-organization in semiconductors and illustrated these by an exemplary model systems which has been in the center of recent theoretical and experimental research. Investigations in diverse semiconductor materials and structures have led to an advanced understanding of the complex dynamics of current filaments in one and two spatial dimensions.

Inspite of the different microscopic nature of those systems some general principles of pattern formation and nonlinear spatio-temporal dynamics in semiconductors can be worked out. Considering the semiconductor as an active medium which is governed by activator-inhibitor kinetics provides such a useful concept. Impact ionization of hot charge carriers plays the role of an activating autocatalytic process not only in the regime of low-temperature impurity breakdown, but is also important in multilayered semiconductor structures. The voltage across the device acts as an inhibitor, and global constraints resulting in a winnertakes-all dynamics are introduced by the external load.

A modeling approach has been described which combines different levels of transport theory, and allows for a detailed understanding of both the microscopic scattering processes in the regime of impurity impact ionization breakdown and the macroscopic dynamics of the filaments for different two-dimensional (rectangular or circular) geometries.

\section{ACKNOWLEDGEMENTS}

I would like to thank C. Lehmann, F.-J. Niedernostheide, V. Novák, W. Prettl, A. Reimann, P. Rodin, and G. Schwarz for stimulating discussions and collaboration.

\section{References}

[1] E. Schöll, Nonequilibrium Phase Transitions in Semiconductors (Springer, Berlin, 1987).

[2] M. P. Shaw, V. V. Mitin, E. Schöll and H. L. Grubin, The Physics of Instabilities in Solid State Electron Devices (Plenum Press, New York, 1992).

[3] B. S. Kerner and V. V. Osipov, Autosolitons (Kluwer Academic Publishers, Dordrecht, 1994).

[4] Nonlinear Dynamics and Pattern Formation in Semiconductors and Devices, edited by F.-J. Niedernostheide (Springer, Berlin, 1995).

[5] E. Schöll, F.-J. Niedernostheide, J. Parisi, W. Prettl and H. Purwins, in Evolution of structures in dissipative continuous systems, edited by F. H. Busse and S. C. Müller (Springer, Berlin, 1998).

[6] Theory of Transport Properties of Semiconductor Nanostructures, Vol. 4 of Electronic Materials Series, edited by E. Schöll (Chapman and Hall, London, 1998).

[7] A. Wacker and E. Schöll, J. Appl. Phys. 78, 7352 (1995).

[8] B. K. Ridley, Proc. Phys. Soc. 82, 954 (1963).

[9] K. Mayer, J. Parisi and R. Huebener, Z. Phys. B 71, $171(1988)$

[10] A. Wierschem, F.-J. Niedernostheide, A. Gorbatyuk and H.-G. Purwins, Scanning 17, 106 (1995).

[11] A. Brandl, M. Völcker and W. Prettl, Appl. Phys. Lett. 55, 238 (1989).

[12] J. Spangler, B. Finger, C. Wimmer, W. Eberle and W. Prettl, Semicond. Sci. Technol. 9, 373 (1994).

[13] B. Kukuk, F. Reil, F.-J. Niedernostheide and H.G. Purwins, in Selforganization in Activator-InhibitorSystems, edited by H. Engel, F.-J. Niedernostheide, H. G. Purwins and E. Schöll (Wissenschaft \& Technik Verlag, Berlin, 1996), pp. 62-66.

[14] H. Baumann, R. Symanczyk, C. Radehaus, H. Purwins and D. Jäger, Phys. Lett. A 123, 421 (1987).

[15] F.-J. Niedernostheide, M. Arps, R. Dohmen, H. Willebrand and H.-G. Purwins, phys. status solidi (b) 172, 249 (1992).

[16] W. Eberle, J. Hirschinger, U. Margull, W. Prettl, V. Novák and H. Kostial, Appl. Phys. Lett. 68, 3329 (1996). 
[17] Nonlinear and Chaotic Transport Phenomena in Semiconductors, Special issue of Applied Phys. A 48, 93191, edited by Y. Abe (1989).

[18] E. Schöll, in Handbook on Semiconductors, 2nd ed., edited by P. T. Landsberg (North Holland, Amsterdam, 1992), Vol. 1.

[19] A. Wacker and E. Schöll, Semicond. Sci. Technol. 9, 592 (1994).

[20] R. Stasch, R. Hey, M. Asche, A. Wacker and E. Schöll, J. Appl. Phys. 80, 3376 (1996).

[21] A. Alekseev, S. Bose, P. Rodin and E. Schöll, Phys. Rev. E 57, 2640 (1998).

[22] F.-J. Niedernostheide, B. S. Kerner and H.-G. Purwins, Phys. Rev. B 46, 7559 (1992).

[23] M. Meixner, P. Rodin and E. Schöll, Phys. Rev. E 58, 2796 (1998).

[24] M. Meixner, P. Rodin and E. Schöll, Phys. Rev. E 58, 5586 (1998).

[25] Negative Differential Resistance and Instabilities in two-dimensional Semiconductors, edited by N. Balkan, B. K. Ridley and A. J. Vickers (Plenum Press, New York, 1993).

[26] Z. S. Gribnikov, K. Hess and G. Kosinovsky, J. Appl. Phys. 77, 1337 (1995).

[27] L. L. Bonilla, J. Galán, J. A. Cuesta, F. C. Martínez and J. M. Molera, Phys. Rev. B 50, 8644 (1994).

[28] F. Prengel, A. Wacker and E. Schöll, Phys. Rev. B 50, 1705 (1994), ibid 52, 11518 (1995).

[29] H. T. Grahn, in Semiconductor Superlattices, Growth and Electronic Properties, edited by H. T. Grahn (World Scientific, Singapore, 1995), Chap. 4.

[30] A. Wacker, in Theory of transport properties of semiconductor nanostructures, edited by E. Schöll (Chapman and Hall, London, 1998), Chap. 10.

[31] F. Piazza, P. C. M. Christianen and J. C. Maan, Phys. Rev. B 55, 15591 (1997).

[32] Quantum Transport in Ultrasmall Devices, edited by D. K. Ferry, L. Grubin, C. Jacoboni and A. P. Jauho (Plenum Press, New York, 1995).

[33] K. Aoki, T. Kobayashi and K. Yamamoto, J. Physique Colloque C 7, 51 (1981).

[34] S. W. Teitsworth, R. M. Westervelt and E. E. Haller, Phys. Rev. Lett. 51, 825 (1983).

[35] E. Schöll and D. Drasdo, Z. Phys. B 81, 183 (1990).

[36] R. E. Kunz and E. Schöll, Z. Phys. B 89, 289 (1992).

[37] G. Hüpper, K. Pyragas and E. Schöll, Phys. Rev. B 47, 15515 (1993).

[38] G. Hüpper, K. Pyragas and E. Schöll, Phys. Rev. B 48, 17633 (1993).

[39] R. E. Kunz and E. Schöll, Z. Phys. B 99, 185 (1996).
[40] M. Gaa and E. Schöll, Phys. Rev. B 54, 16733 (1996).

[41] V. Novák, C. Wimmer and W. Prettl, Phys. Rev. B 52, 9023 (1995).

[42] T. Kuhn, G. Hüpper, W. Quade, A. Rein, E. Schöll, L. Varani and L. Reggiani, Phys. Rev. B 48, 1478 (1993).

[43] W. Quade, G. Hüpper, E. Schöll and T. Kuhn, Phys. Rev. B 49, 13408 (1994).

[44] B. Kehrer, W. Quade and E. Schöll, Phys. Rev. B 51, 7725 (1995)

[45] H. Kostial, M. Asche, R. Hey, K. Ploog, B. Kehrer, W. Quade and E. Schöll, Semicond. Sci. Technol. 10, 775 (1995).

[46] M. Gaa, R. E. Kunz and E. Schöll, in Proc. 9th Int. Conf. on Hot Carriers in Semiconductors, edited by K. Hess, J. P. Leburton and U. Ravaioli (Plenum, New York, 1996), pp. 347-351.

[47] M. Gaa, R. E. Kunz and E. Schöll, Phys. Rev. B 53, 15971 (1996)

[48] K. Kunihiro, M. Gaa and E. Schöll, Phys. Rev. B 55, 2207 (1997).

[49] G. Schwarz and E. Schöll, Acta Technica CSAV 42, 669 (1997).

[50] G. Schwarz, C. Lehmann and E. Schöll, in Proc. 24th International Conference on The Physics of Semiconductors (ICPS-24), Jerusalem, Israel, edited by D. Gershoni (World Scientific, Singapore, 1999).

[51] E. Schöll, Z. Phys. B 46, 23 (1982).

[52] R. E. Kunz, E. Schöll, H. Gajewski and R. Nürnberg, Sol. State El. 39, 1155 (1996).

[53] M. Gaa, R. E. Kunz, E. Schöll, W. Eberle, J. Hirschinger and W. Prettl, Semicond. Sci. Technol. 11, 1646 (1996).

[54] A. Brandl, T. Geisel and W. Prettl, Europhys. Lett. 3, 401 (1987).

[55] A. Brandl, W. Kröninger, W. Prettl and G. Obermair, Phys. Rev. Lett. 64, 212 (1990).

[56] U. Rau, W. Clauss, A. Kittel, M. Lehr, M. Bayerbach, J. Parisi, J. Peinke and R. Huebener, Phys. Rev. B 43 , 2255 (1991).

[57] G. Hüpper and E. Schöll, Phys. Rev. Lett. 66, 2372 (1991).

[58] J. Hirschinger, F. J. Niedernostheide, W. Prettl, V. Novák, M. Cukr, J. Oswald and H. Kostial, Acta Techn. CSAV 42, 661 (1997).

[59] J. Hirschinger, W. Eberle, W. Prettl, F.- J. Niedernostheide and H. Kostial, Phys. Lett. A 236, 249 (1997).

[60] L. Schimansky-Geier, C. Zülicke and E. Schöll, Z. Phys. B 84, 433 (1991). 